\title{
Diseño experimental de Aerogenerador tipo Savonius
}

\section{Experimental design of Savonius wind turbine}

\author{
SANCHEZ-CORTEZ, Jose Alfonso, CHAVEZ-CASTILLO, José Giovann, SANCHEZ-GUTIERREZ, \\ Carlos Ernesto y TRINIDAD-SULVARAN, Ingrid Aranxa
}

Universidad Tecnologica de Altamira

ID 1 ${ }^{\mathrm{er}}$ Autor: Jose Alfonso, Sanchez-Cortez / CVU CONACYT ID: 500152

ID $1^{\text {er }}$ Coautor: José Giovann, Chavez-Castillo / ORC ID: 0000-0003-1464-9815

ID $2^{\text {do }}$ Coautor: Carlos Ernesto, Sanchez-Gutierrez, / ORC ID: 0000-0002-9636-9018

ID $3^{\text {er }}$ Coautor: Ingrid Aranxa, Trinidad-Sulvaran / ORC ID: 0000-0002-8222-4651

\section{Resumen}

Este proyecto está relacionado con la tecnología en energías renovables, se describe el cálculo y el diseño de un aerogenerador de eje vertical tipo Savonius experimental. Se tiene como objetivo la adquisición de información necesaria para la construcción y modelado de un aerogenerador experimental. Con los resultados obtenidos, se podría llegar a tener datos como velocidad de viento e información para modificar la construcción del prototipo de acuerdo con estas características y cubrir así las necesidades energéticas con las que se cuenta. Se realizó un amplio estudio en donde se obtuvo el recurso eólico con el que se cuenta en la zona en la que se va a aplicar, debido a que el estudio de velocidad del viento proviene del lugar de instalación. Los cálculos realizados incluyen la generación de potencia eléctrica aprovechable a partir de la velocidad del viento y la velocidad a la que puede llegar a girar el dispositivo.

\section{Savonious, Aerogenerador, Energías Renovables}

\begin{abstract}
This project applies renewable energy technology, the calculation and design of an experimental Savonius vertical axis wind turbine is described. The objective is to acquire the necessary information for the construction and modeling of an experimental wind turbine. This prototype was constructed to use the movement of the air to generate electricity in a clean and non-contaminant way. The parts were assembled to make as much energy as its possible, those designs were calculated using the formulas of wind resource harnessing. Following this clean and non contaminant ideas, we recycled most of the materials used for the construction of this prototype. We calculated the rotative speed of the device according to the wind, we also calculated the electric energy that the device could generate with the wind. We studied the environment data such as wind speed, temperature for three weeks, where installed the experimental device it is going to be.
\end{abstract}

Renewable Energies, Wind Turbine, Savonious

\footnotetext{
* Correspondencia del Autor (jsanchez@utaltamira.edu.mx)

$\dagger$ Investigador contribuyendo como primer autor.
} 


\section{Introducción}

El Diseño Experimental de Aerogenerador Tipo Savonius, es un prototipo modelado a escala por los alumnos de ingeniería del 8vo cuatrimestre en Energías Renovables de la Universidad Tecnológica de Altamira. Este proyecto es desarrollado con la finalidad de presentar una alternativa al estudio de la energía eólica durante el periodo de educación de alumnos de nivel medio superior y superior, el cual consta de un modelo a escala de un generador eólico tipo Savonius.

El prototipo, constara con un sistema de medición de velocidad del viento, rotaciones por minuto (RPM) y generación de energía, para que, con estos datos generados por medio de un sensor de Efecto Hall conectado a un Arduino, llevar a cabo prácticas con mayor valor interactivo hacia los estudiantes y de este modo comparar con mayor facilidad los datos recaudados del prototipo con los datos generados en el aula durante clase.

\section{Antecedentes}

Un molino es una máquina que transforma el viento en energía aprovechable, que proviene de la acción de la fuerza del viento sobre unas aspas oblicuas unidas a un eje común. El eje giratorio puede conectarse a varios tipos de maquinaria para moler grano, bombear agua o generar electricidad. Cuando el eje se conecta a una carga, como una bomba, recibe el nombre de molino de viento. Si se usa para producir electricidad se le denomina generador de turbina de viento. Los molinos tienen un origen remoto. (Voneschen, 2017)

La referencia más antigua que se tiene es un molino de viento que fue usado para hacer funcionar un órgano musical en el siglo I era común. Los primeros molinos de uso práctico fueron construidos en Sistan, Afganistán, en el siglo VII. Estos fueron molinos de eje vertical con hojas rectangulares (Voneschen, 2017). Aparatos hechos de 6 a 8 velas de molino cubiertos con telas fueron usados para moler cereales o extraer agua.

\section{Siglo I D.C.}

Herón de Alejandría inventa el primer molino de viento y se gana un lugar en la historia. Herón, aprovechó el poder del viento para accionar un órgano musical.
Las aspas del molino, estaban unidas a un eje que impulsaba unos pistones hacia arriba y hacia abajo para producir sonidos con el órgano.

\section{Siglo IX D.C.}

Los persas construyen un molino de viento que posiblemente era utilizado para bombear agua $o$ moler maíz. Las velas verticales con las que se accionaba el molino, eran probablemente hechas de caña y madera. Las velas se encontraban al interior de una construcción amurallada con una puerta abierta en el frente, que guiaba el viento a través de la máquina.

\section{Siglo XII D.C.}

En este siglo ya se construyen molinos de viento en Europa. Este tipo de molino de viento se fabricaba sobre un poste vertical, con un sistema rotacional de manera que el molino pudiera moverse con la dirección del viento. Estos molinos verticales eran comúnmente utilizados para moler maíz o bombear agua.

\section{Siglo XIII D.C.}

Los molinos de torre, fueron el diseño más novedoso en el oeste de Europa durante este siglo. Estos molinos se construían en grandes torres de piedra, tenían aspas y un rotor montado en el techo de la torre. Se colocaba también una veleta detrás de las aspas, para guiarlas en la dirección del viento.

\section{Siglo XIX D.C.}

En 1887, el inventor estadounidense Charles Brush, construyó la primera turbina eólica automática. Se le llamó "turbina eólica" por primera vez y no "molino de viento" ya que se utilizaba para generar electricidad. La turbina eólica pesaba 4 toneladas y alimentaba a un generador de $12 \mathrm{~kW}$ de potencia.

\section{2}

La turbina eólica Savonius, es una turbina de ejes verticales inventada por el ingeniero finlandés Sigurd Johannes Savonius. La turbina Savonius es un dispositivo de arrastre, es decir que la energía rotacional se genera por el empuje del viento y no por sustentación aerodinámica, como la mayoría de las turbinas modernas. 


\section{7}

La turbina eólica Giromill, es una turbina de eje vertical con diseño Darrieus. La turbina Giromill utiliza sustentación en vez de arrastre y es habitualmente fabricada con dos o tres aspas que rotan alrededor de un eje central. La turbina Giromill es una turbina de baja eficiencia ya que necesita de vientos de alta velocidad para comenzar a rotar.

\section{1}

La turbina eólica Darrieus es también conocida como la turbina "batidora de huevos". Inventada por el ingeniero francés Georges Jean Marie Darrieus. El diseño de esta turbina usualmente consta de dos aspas que giran en torno a una torre de eje vertical, con un generador eléctrico cerca de la base de la máquina.

\section{1}

En 1941 la turbina eólica Smith-Putnam, fue la primera turbina eólica de tamaño megawatt. Era una turbina con dos aspas de 53 metros de diámetro y construida sobre una torre de celosía de 36 metros de alto. Sólo operó por 1100 horas, hasta que una de las aspas falló.

\section{Turbina eólica moderna}

La turbina eólica moderna, es el resultado de años de desarrollo e investigación científica. Hoy en día, la mayoría de las turbinas modernas provienen de la familia de las turbinas con ejes horizontales y tienen 3 aspas. Las aspas o palas de las turbinas eólicas, tienen un diseño similar a las alas de los aviones y utilizan el sustento aerodinámico del viento para rotar. (Eolicos, 2018)

Las turbinas modernas fueron desarrolladas a comienzos de 1980, si bien, los diseños continúan en desarrollo. La industria de la energía eólica en tiempos modernos comenzó en 1979 con la producción en serie de turbinas de viento por los fabricantes Kuriant, Vestas, Nordtank, y Bonus. Aquellas turbinas eran pequeñas para los estándares actuales, con capacidades de 20 a $30 \mathrm{~kW}$ cada una. Desde entonces, la talla de las turbinas ha crecido enormemente, y la producción se ha expandido a muchos países. (Voneschen, 2017).

\section{Objetivo}

\section{Objetivo General}

Diseñar un prototipo a escala de un aerogenerador Tipo Savonious que sirva de herramienta didáctica para ser utilizado por alumnos de educación media superior y superior, así como realizar la toma de datos cuantificables, como lo son la velocidad del viento, velocidad de rotación del prototipo y generación eléctrica estimada entre otros, mostrando así la importancia de la captura de datos para la instalación de un equipo a grande escala.

\section{Objetivos Particulares.}

Diseño y construcción de un modelo a escala de un aerogenerador tipo Savonius, para utilizarlo con fines didácticos en un ambiente medio superior y superior.

- $\quad$ Promover la práctica de las estimaciones y cálculo de generación eléctrica para explotar la fuerza del viento y sus efectos en el aerogenerador dentro de los ambientes educativos, evitando así la instalación innecesaria o desaprovechamiento del recurso eólico.

\section{Justificación}

Proveer a el alumnado de educación media y superior, de un equipo didáctico capaz de enseñar de una manera más fácil y eficiente, el funcionamiento de un aerogenerador de eje vertical tipo Savonius, explicando desde la construcción de mismo hasta su funcionamiento interno, para la medición de datos.

\section{Marco teórico}

Los aerogeneradores producen electricidad aprovechando la energía natural del viento para impulsar un generador. El viento es una fuente de energía limpia, sostenible que nunca se agota, y la transformación de su energía cinética en energía eléctrica no produce emisiones. (Sanchez, 2017). A finales del siglo XIX aparecen las primeras turbinas eólicas para generar electricidad. Pero no fue hasta después de la II Guerra Mundial, cuando una compañía danesa empezó a fabricar las primeras turbinas de corriente alterna. 
Sin embargo, el gran interés por esta energía renovable, vino con la primera crisis del petróleo (1973), y en los años 80, surgió el boom industrial y tecnológico para los generadores modernos. de 3 palas:

Elementos básicos de un aerogenerador

Góndola: Contiene los componentes clave del aerogenerador, incluyendo el multiplicador y el generador eléctrico. El personal de servicio puede entrar en la góndola desde la torre de la turbina. A la izquierda de la góndola se en el rotor del aerogenerador, es decir las palas y el buje. (Sanchez, 2017)

Palas del rotor: Capturan el viento y transmiten su potencia hacia el buje. En un aerogenerador moderno de $600 \mathrm{~kW}$, cada pala mide alrededor de 20 metros de longitud y su diseño es muy parecido al del ala de un avión. (Sanchez, 2017)

Buje: El buje del rotor está acoplado al eje de baja velocidad del aerogenerador. (Sanchez, 2017)

Eje de baja velocidad: Conecta el buje del rotor al multiplicador. En un aerogenerador moderno de $600 \mathrm{~kW}$ el rotor gira muy lento, aproximadamente de 19 a 30 revoluciones por minuto (r.p.m.) El eje contiene conductos del sistema hidráulico para permitir el funcionamiento de los frenos aerodinámicos. (Sanchez, 2017)

Multiplicador: Tiene a su izquierda el eje de baja velocidad. Permite que el eje de alta velocidad que está a su derecha gire 50 veces más rápido que el eje de baja velocidad. (Sanchez, 2017)

Eje de alta velocidad: Gira aproximadamente a 1.500 r.p.m. lo que permite el funcionamiento del generador eléctrico. Está equipado con un freno de disco mecánico de emergencia. El freno mecánico se utiliza en caso de fallo del freno aerodinámico, o durante las labores de mantenimiento de la turbina. (Sánchez, 2017)

Generador eléctrico: Suele ser un generador asíncrono o de inducción. En los aerogeneradores modernos la potencia máxima suele estar entre 500 y $1.500 \mathrm{~kW}$. (Sanchez, 2017)
Controlador electrónico: Es un ordenador que continuamente monitoriza las condiciones del aerogenerador y que controla el mecanismo de orientación. En caso de cualquier disfunción (por ejemplo, un sobrecalentamiento en el multiplicador $\mathrm{o}$ en el generador), automáticamente para el aerogenerador y llama al ordenador del operario encargado de la turbina a través de un enlace telefónico mediante un modem. (Sanchez, 2017)

Unidad de refrigeración: Contiene un ventilador eléctrico utilizado para enfriar el generador eléctrico. Además, contiene una unidad refrigerante por aceite empleada para enfriar el aceite del multiplicador. Algunas turbinas tienen generadores refrigerados por agua. (Sánchez, 2017)

Torre: Soporta la góndola y el rotor. Generalmente es una ventaja disponer de una torre alta, dado que la velocidad del viento aumenta conforme nos alejamos del nivel del suelo. Una turbina moderna de $600 \mathrm{~kW}$ tendrá una torre de 40 a 60 metros (la altura de un edificio de 13 a 20 plantas). (Leiva, 2017)

Las torres pueden ser bien torres tubulares o torres de celosía. Las torres tubulares son más seguras para el personal de mantenimiento de las turbinas ya que pueden usar una escalera interior para acceder a la parte superior de la turbina. La principal ventaja de las torres de celosía es que son más baratas. (Leiva, 2017)

Mecanismo de orientación: Está activado por el controlador electrónico, que vigila la dirección del viento utilizando la veleta. (Sanchez, 2017)

\section{Principio de sustentación}

Para entender el funcionamiento de un aerogenerador, se tiene que comprender este principio, ya que la correcta operación de una turbina eólica es dependiente de dicho principio. (Pablo Bejerano, 2016)

Este principio viene implicado en los alerones o alas de un avión. Esto quiere decir que un ala está hecha en dos partes, la superior y la inferior, donde la velocidad del viento se comporta de manera diferente. En la siguiente imagen se muestran las partes anteriormente mencionadas: 


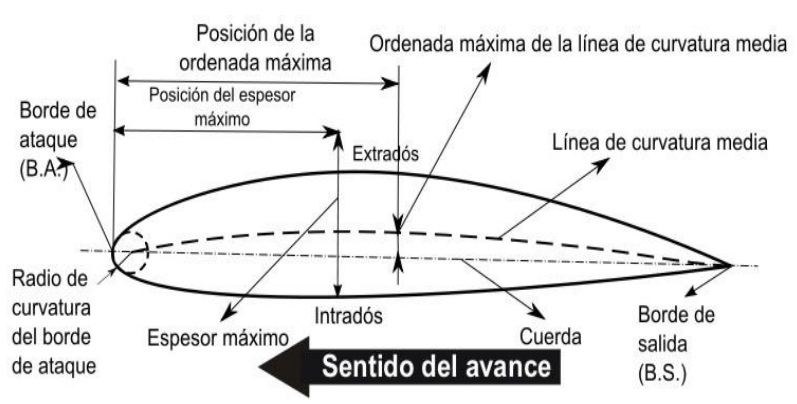

Figura 1 Ala bajo el principio de sustentación

Como se observa en la imagen, tanto el ala de un avión está hecha bajo principios físicos, de la misma manera, las aspas de un aerogenerador. Se pueden derivar las siguientes partes del ala mostrada en la imagen:

Extradós: Es la parte superior del ala (La parte que mira al cielo). Esa parte está más curvada (se menciona la razón más adelante).

- Intradós: Parte inferior del plano (La que mira al suelo). Esta parte tiene menos curvatura.

- $\quad$ Borde de ataque: La parte delantera del plano. Donde primero impactan las partículas de aire.

- $\quad$ Borde de salida o de fuga: Parte trasera del plano.

Cuerda: Línea imaginaria que se forma al unir el borde de ataque con el borde de salida.

Como se aprecia en la imagen, la parte superior e inferior tienen diferente curvatura, lo que hace que el viento se tenga que mover más rápido al cruzar la parte superior por la mayor distancia, y por abajo tendría que ir más lento, por la menor distancia. (Pablo Begerano, 2016) Una vez que se tiene este principio claro, se continúa con el teorema de Bernoulli, que estipula lo siguiente:

Al aumentar la velocidad de las partículas de aire, su presión disminuye y de manera viceversa. De lo que se obtiene:

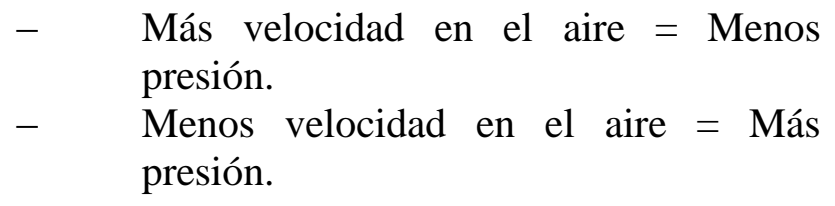

En base a lo anterior se puede concluir que en el extradós (parte superior) el aire va más rápido, por lo que hay menos presión y en el intradós (parte inferior) va más lento, por lo que hay más presión, esto crea una fuerza que empuja al ala hacia arriba. (Pablo Begerano, 2016).

Entendiendo este principio, se comprende que de esta misma manera funciona un aerogenerador, las aspas están hechas de la misma forma, utilizando principios físicos que le permiten girar con facilidad, aún con poca velocidad del viento.(Pablo Begerano, 2016)

\section{Aerogeneradores de Eje Vertical}

Como su nombre lo indica, este tipo de aerogeneradores tienen la característica de que sus palas rotan en torno a un eje vertical, lo que presenta tres ventajas fundamentales con respecto a los aerogeneradores de eje horizontal:

- $\quad$ La sujeción de las palas es de fácil diseño y ejecución.

- $\quad$ No precisan sistema de orientación para captar la energía del viento.

Fácil ubicación del tren de potencia, generador y transformador, a nivel del suelo.

Como se mencionó anteriormente, este tipo de aerogeneradores no necesitan mecanismo de orientación, a pesar de esto su producción energética es menor y dependiendo del tipo de aerogenerador puede necesitar un motor para romper la inercia y se le pueda poner en marcha. De los aerogeneradores verticales se derivan tres tipos:

\section{Savonius:}

Se caracteriza por tener dos semicírculos desplazados horizontalmente a una determinada distancia, a través de la cual el aire es desplazado, por lo que tiene una pequeña producción energética. (Structuralia, 2018).

\section{Darieus:}

Este tipo de aerogenerador está compuesto por dos o más palas biconvexas que se unen al eje vertical tanto por la parte superior e inferior, lo que permite el aprovechamiento de una alta gama de velocidades del viento, pero para poder girar necesitan un tipo de rotor Savonius. (Structuralia, 2018).

SANCHEZ-CORTEZ, Jose Alfonso, CHAVEZ-CASTILLO, José Giovann, SANCHEZ-GUTIERREZ, Carlos Ernesto y TRINIDADSULVARAN, Ingrid Aranxa. Diseño experimental de Aerogenerador tipo Savonius. Revista de Sistemas Experimentales. 2019 


\section{Giromill:}

Tiene un conjunto de palas verticales unidas con dos barras en el eje vertical y ofrece un rango de suministro energético de 10 a $20 \mathrm{~kW}$. (Structuralia, 2018).

En palabras generales se concluye que el rendimiento de los aerogeneradores de eje vertical se encuentra por debajo de la mitad que el de su competencia directa, que son los de eje horizontal, hecho que ha empujado a las industrias a enfocar su atención a los del segundo caso (eje horizontal). (Sanchez, 2017)

\section{Desarrollo}

\section{Cálculos involucrados en el diseño}

En primer lugar, se tiene que considerar el cálculo del viento y estimación aproximada de posible potencia en función del viento. Esto se hace con un anemómetro, que es un dispositivo capaz de registrar velocidades del viento, dependiendo del modelo también puede medir temperatura, presión, entre otros parámetros necesarios que se deben considerar para la colocación de un aerogenerador. (Roman, 2018)

\section{Estudio del viento}

En el estudio llevado a cabo en la Universidad Tecnológica de Altamira, registramos los siguientes valores de velocidad media por día del viento por 14 días.

\begin{tabular}{|l|r|}
\hline Día & Velocidad Registrada en $\mathrm{m} / \mathrm{s}$ \\
\hline Lunes & 8.61 \\
\hline Martes & 12.5 \\
\hline Miércoles & 7.77 \\
\hline Jueves & 6.11 \\
\hline Viernes & 7.77 \\
\hline Sábado & 13.05 \\
\hline Domingo & 3.47 \\
\hline Lunes & 12.4 \\
\hline Martes & 4.3 \\
\hline Miércoles & 7.16 \\
\hline Jueves & 12.1 \\
\hline Viernes & 4.9 \\
\hline Sábado & 10.23 \\
\hline
\end{tabular}

De la tabla anterior, se obtuvo la conclusión que generalmente se tiene un buen recurso eólico que no se aprovecha. Se muestra en la siguiente gráfica el comportamiento del viento obtenido.

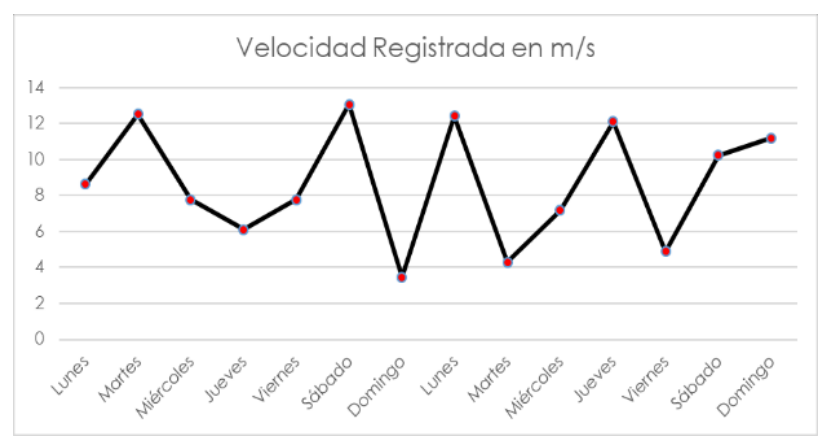

\section{Cálculo de potencia}

Una vez obtenidos los valores anteriores se procede en obtener el cálculo aproximado obtenido del límite de Betz (en el que explica la obtención de la potencia estimada a partir de la velocidad del viento y del diámetro del aerogenerador). La fórmula dice que en condiciones ideales se puede obtener la siguiente potencia:

$P=(0.29)\left(D^{2}\right)\left(V^{3}\right)$

Donde:

(0.29): Es una constante.

D: Es el diámetro del aerogenerador.

$\mathrm{V}$ : Es la velocidad del viento.

Pero como sabemos, las condiciones ideales no existen, como se dio cuenta el científico alemán Betz, por lo que desarrolló una formula restando el posible desaprovechamiento completo del viento, la resistencia al aire de las aspas y que los rodamientos no giran a la máxima velocidad aprovechable.

$P=(0.15)\left(D^{2}\right)\left(V^{3}\right)$

Para obtener el valor del viento, obtuvimos la media de los datos colectados por los 14 días, dando un valor de $\mathbf{8 . 6 8} \mathbf{~ m} / \mathbf{s}$. Sustituyendo en la formula, se obtiene que obtendríamos un valor aproximado de $\mathbf{0 . 1 3 6 3}$ W. De lo que concluimos es una buena cantidad de potencia. (Roman, 2018)

\section{Cálculo de velocidad de giro de una eólica}

Este cálculo es para aproximar a cuanta velocidad en rpm giraría la turbina eólica. La fórmula estipula lo siguiente:

$n=\frac{(60)(\lambda)(v)}{(\pi)(D)}$ 
Donde:

$\mathrm{N}=$ es el número de revoluciones por minuto [rpm].

$\lambda=$ se llama velocidad específica y se denomina (TSR). Este factor depende del tipo de eólica (rápida o lenta). Puede tener un valor comprendido entre aprox. 0.80 y 10. (Se le asigna un valor de 0.9 ).

$\mathrm{v}=$ es la velocidad del viento en metros por segundo $[\mathrm{m} / \mathrm{s}]$.

$\mathrm{D}=$ es el diámetro de la eólica en metros [m].

$\pi=$ es pi $(3,1415926)$.

La fórmula se muestra con nuestros datos:

$n=\frac{(60)(0.9)(8.68 \mathrm{~m} / \mathrm{s})}{(\pi)(0.260 \mathrm{~m})}=\mathbf{5 7 3 . 8 3} \boldsymbol{R P} \boldsymbol{M}$

Lo que dice que a una velocidad promedio giraría bastante rápido considerando las características del aerogenerador. Se tiene en cuenta que la velocidad del viento varía conforme a la altura como se aprecia en la siguiente imagen.
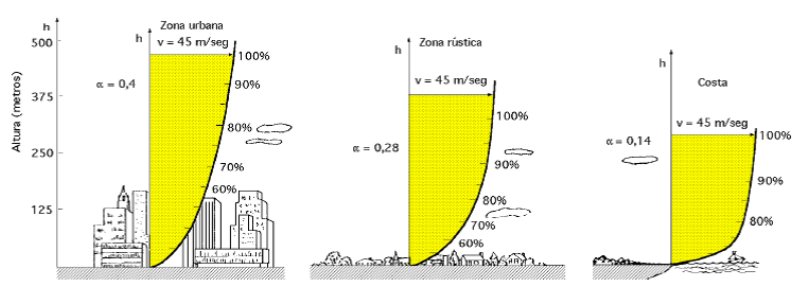

Figura 3 Se muestran las variaciones del viento con respecto a la altura

(Quintana, 2015)

\section{Curva de potencia}

La curva de potencia se calculó en base a los datos obtenidos de la velocidad del viento, como se muestra en la siguiente tabla:

\begin{tabular}{|r|r|}
\hline Potencia en watts & Velocidad del viento en $\mathbf{~} / \mathbf{s}$ \\
\hline 0.0157 & 1 \\
\hline 0.0314 & 2 \\
\hline 0.0471 & 3 \\
\hline 0.0628 & 4 \\
\hline 0.0785 & 5 \\
\hline 0.0942 & 6 \\
\hline 0.1099 & 7 \\
\hline 0.1256 & 8 \\
\hline 0.1413 & 9 \\
\hline 0.1570 & 10 \\
\hline 0.1727 & 11 \\
\hline 0.1884 & 12 \\
\hline 0.2041 & 13 \\
\hline 0.2198 & 14 \\
\hline 0.2355 & 15 \\
\hline 0.2512 & 16 \\
\hline 0.2669 & 17 \\
\hline 0.2826 & 18 \\
\hline 0.2984 & 19 \\
\hline 0.3141 & 20 \\
\hline &
\end{tabular}

Con su respectiva gráfica:

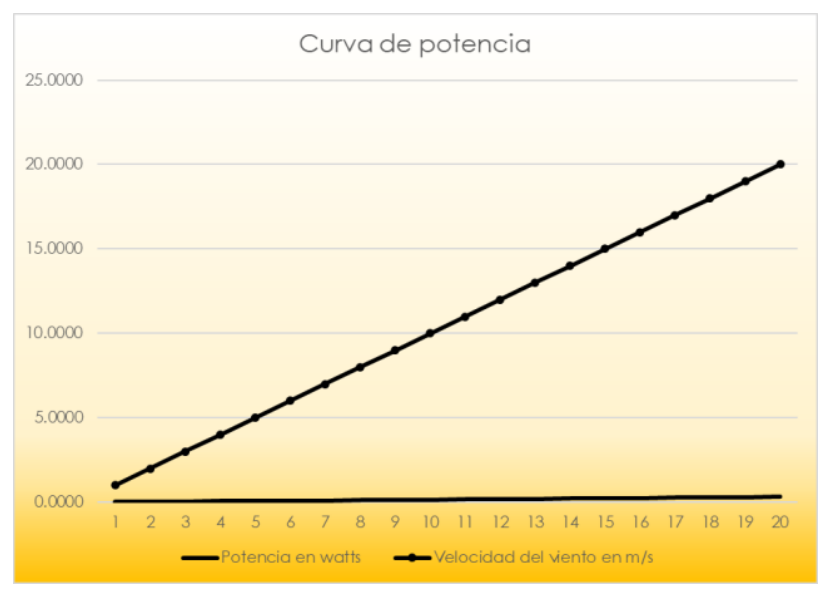

(Navarra, 2015)

\section{Diseño de palas}

Se sabe que para un aerogenerador con 3 aspas se debe tener un espacio de $120^{\circ}$ entre cada pala, pero a pesar de esto, se tiene que calcular el diseño de las mismas, por su perfil aerodinámico, para su mejor aprovechamiento del viento. Para esto se buscan fórmulas de diseño aerodinámico, debido a su diseño no plano, si no helicoidal (esto quiere decir curveado como se muestra en la siguiente imagen).

Se escogió este tipo de aerogenerador debido a la sencillez y eficiencia en base a su diseño, en la siguiente imagen se aprecian los rendimientos de cada tipo de aerogenerador:

Para empezar, se debe asignar un coeficiente de solidez, para definir el control de la maquina a velocidades de viento, se considera que este valor debe encontrarse entre $0.20-0.40$ debido a que para valores menores existe un mayor punto de descontrol, lo que dificulta el arranque y desplaza el coeficiente de potencia a valores de TSR (Tip Speed Ratio) más elevados.

El BAR (aspecto de álabe) es un parámetro importante que define la longitud de la cuerda (c) y la longitud del álabe (1). Cabe mencionar que los efectos de flujo tridimensional sobre cada sección del perfil aerodinámico tienden a despreciarse. Esto se calcula de la siguiente manera:

$B A R=\frac{(L)}{(c)}$ 
Donde:

$\mathrm{C}=$ Cuerda del perfil $(\mathrm{m})$

$\mathrm{L}=$ Longitud de los álabes $(\mathrm{m})$

Con estos datos podemos calcular el número de Reynolds, de esto depende la fineza de un ala. Este número es una medida de la calidad de circulación del aire, alrededor de la misma y que se define de la siguiente manera:

$R e=\frac{(v)(t)}{(v)}$

Donde:

$\mathrm{t}=$ Anchura del ala (Longitud de la cuerda del perfil. $\mathrm{m}$ )

$\mathrm{v}=$ Velocidad del aire que la barre $\left(\mathrm{m}^{2} / \mathrm{s}\right)$

$v=$ Viscocidad cinemática del aire bajo condiciones normales $=15 \times 10^{-6}\left(\mathrm{~m}^{2} / \mathrm{s}\right)$

Pero toda ala tiene su número de Reynolds crítico $\left(R e_{\text {crit }}\right)$. Si la medida de circulación del aire alrededor del ala es inferior a este valor crítico, el coeficiente de sustentación disminuirá, lo que resultaría en un aumento en la resistencia. En la siguiente imagen se muestra la relación de lo anteriormente estipulado:

La solidez, representa la relación de áreas entre la comprendida por los álabes del rotor, respecto del área total de barrido. Calculado de la siguiente manera:

$\sigma=\frac{(N)(c)(L)}{(A)}=\frac{(N)(c)}{(D)} \sigma=\frac{(N)(c)}{(D)}$

Donde:

$\sigma=$ Solidez

$\mathrm{N}=$ Número e álabes

$\mathrm{C}=$ Cuerda del perfil $(\mathrm{m})$

$\mathrm{D}=$ Diámetro del rotor $(\mathrm{m})$

$\mathrm{L}=$ Longitud de los álabes $(\mathrm{m})$

$\mathrm{A}=$ Área barrida total $\left(\mathrm{m}^{2}\right)$

(Berdugo, 2016)

\section{Agradecimiento}

Un gran agradecimiento a la Universidad Tecnológica de Altamira por el apoyo brindado, para la realización de este proyecto, ya que sin su apoyo mucho de esto no sería posible.

\section{Conclusiones}

El diseño de un prototipo a escala de un aerogenerador tipo savonius puede representar la importancia de la captura de datos cuantificables en el caso de un proyecto de gran magnitud como lo es un parque eólico de equipos con las mismas características.

El diseño de este proyecto puede brindar fácilmente un mejor entendimiento sobre el funcionamiento tanto interno como externo de un aerogenerador tipo savonius, siendo el mismo, un prototipo de bajo costo, que podría estar al alcance de cualquier institución educativa.

El equipo realizado fue hecho a partir de materiales reciclados como tuberías de PVC, estructuras metálicas como soportes, sensor tipo hall, activado por un imán, de esta manera se contabiliza la cantidad de rotaciones que se hacen.

\section{Referencias}

Berdugo, O. I. (2016). Diseño y construcción de un prototipo de turbina eólica de eje vertical para generación a baja potencia.

Caita, C. E. (2014). Diseño y construcción de mini aerogenerador de eje vertical.

Eólicos, G. (2018). Evolución de las turbinas eólicas. Obtenido de Venta de generadores eólicos.

Leiva, M. A. (2017). Diseño de una turbina eólica de eje vertical con rotor mixto.

Navarra, D. (2015). Diseño de un aerogenerador de eje vertical Tipo Savonius. Buenos Aires, Argentina.

Quintana, D. M. (2015). Diseño y cálculo de un aerogenerador de eje vertical.

Román, R. G. (2018). Diseño y calculo preliminar de la torre de un aerogenerador. Madrid, España.

Sánchez, S. d. (2017). Diseño de un aerogenerador tipo H-Dairreus. Valladolid, España.

Voneschen, M. F. (2017). Como construir un generador eolico.

SANCHEZ-CORTEZ, Jose Alfonso, CHAVEZ-CASTILLO, José Giovann, SANCHEZ-GUTIERREZ, Carlos Ernesto y TRINIDADSULVARAN, Ingrid Aranxa. Diseño experimental de Aerogenerador tipo Savonius. Revista de Sistemas Experimentales. 2019 